\title{
MIMO Antenna Design and Channel Modeling
}

\author{
Wenhua Chen, ${ }^{1}$ Manos M. Tentzeris, ${ }^{2}$ Yuan Yao, ${ }^{3}$ Yan Zhang, ${ }^{1}$ and Li Yang ${ }^{4}$ \\ ${ }^{1}$ Department of Electronic Engineering, Tsinghua University, Beijing 100084, China \\ ${ }^{2}$ The School of Electrical and Computer Engineering, Georgia Institute of Technology, Atlanta, GA 30332-0250, USA \\ ${ }^{3}$ School of Electronic Engineering, Beijing University of Posts and Telecommunications, Beijing 100876, China \\ ${ }^{4}$ High-Performance Analog Division, Texas Instruments, Dallas, TX 75266-0199, USA
}

Correspondence should be addressed to Wenhua Chen, chenwh@tsinghua.edu.cn

Received 31 October 2012; Accepted 31 October 2012

Copyright (C) 2012 Wenhua Chen et al. This is an open access article distributed under the Creative Commons Attribution License, which permits unrestricted use, distribution, and reproduction in any medium, provided the original work is properly cited.

The multiple-input multiple-output (MIMO) wireless technique represents a breakthrough in the use of antenna arrays in wireless systems. MIMO technology has attracted attention in wireless communications, because it offers significant increases in data throughput and link range without additional bandwidth or increased transmit power. It achieves this goal by spreading the same total transmit power over the antennas to achieve an array gain that improves the spectral efficiency (more bits per second per hertz of bandwidth) or to achieve a diversity gain that improves the link reliability (reduced fading). As demonstrated in Figures 1(a) and 1(b), the research topic on MIMO antenna design has become a quite popular field in wireless communication area.

Unlike traditional phased arrays or diversity techniques that improve the sensitivity to one signal of interest, MIMO systems employ antenna arrays jointly at transmit and receive sides to spatially multiplex signals over multipath or near-field channels. The design of MIMO antennas and propagation channels will affect the channel correlation significantly, and this will in turn determine the system capacity. In a multipath-rich environment, it can utilize uncorrelated channels exploited by multiple antennas at both the transmitter and receiver to convey different signals simultaneously without expanding bandwidth. Antenna design plays an important role in MIMO systems since antenna characteristics, including radiation pattern, polarisation, array arrangement, and mutual coupling, have great impact on the correlation between different signal branches. For small terminal devices such as personal digital assistant (PDAs), MIMO antenna design is more challenging, because the antenna structure is required to be more compact and easily assembled with the device as well as suitable for preserving channel capacity.

As the transmission medium for wireless and mobile communications, radio channel modeling is the fundamental work to the communications systems. The accurate understanding of actual propagation channel is deterministic for algorithm design and optimization, equipment development, system deployment and operation. Channel modeling work also plays an important role in the communication standardization process.

With the continuous and rapid improvement of the communication techniques, the realization of radio channel modeling developed from large scale to small scale, from static to dynamic, and from narrowband to broadband. In recent years, the channel characteristics research went into the more sophisticated spatial area. Multiple-input multipleoutput (MIMO) technology makes the space, together with time and frequency, become a resource improving the performance of wireless communication. With the proposals and application for distributed antennas, large MIMO, and 3D MIMO technologies, the demand for the spatial characteristics of the channel modeling is growing continuously. The coupling relationship between the space, time, and frequency has an important role for the performance of wireless communication systems.

Multilink collaboration will act as the main feature for the future systems. Then multipoint network channel model is increasingly required to provide guides in the basic research field. In the networking collaboration environment, the correlation between the parameters and links, which is 


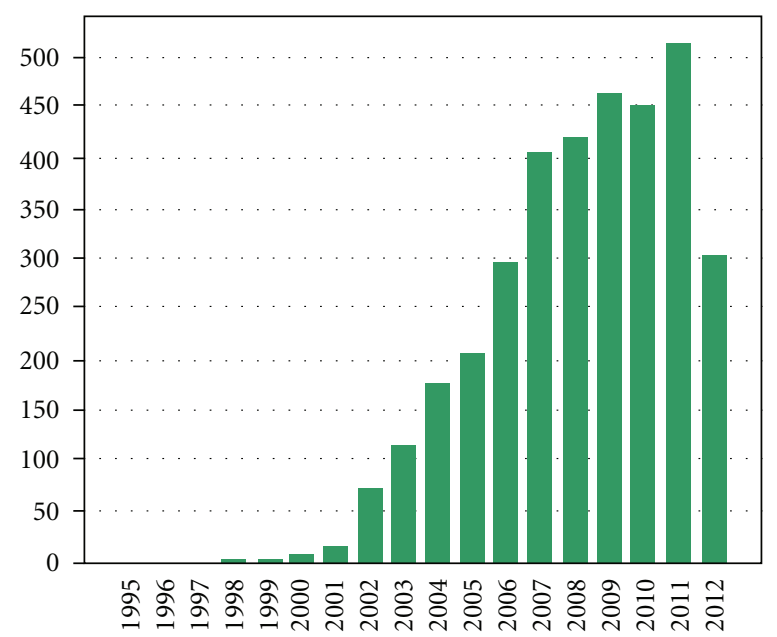

(a)

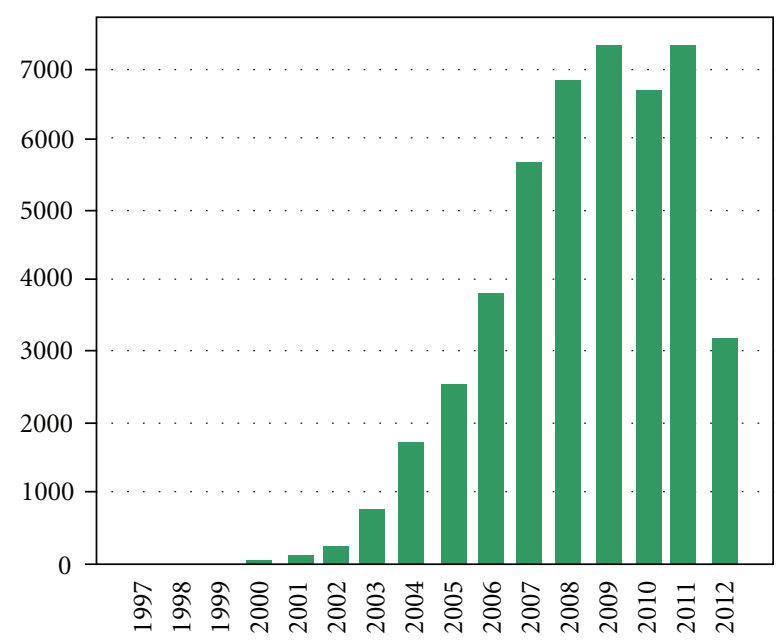

(b)

Figure 1: (a) Paper number published per year. (b) Paper citation per year.

ignored in previous studies, will determine the network's coverage, the reliability, and capacity. These correlations may be the key to solving the future channel modeling problem.

Wenhua Chen

Manos M. Tentzeris

Yuan Yao

Yan Zhang

Li Yang 

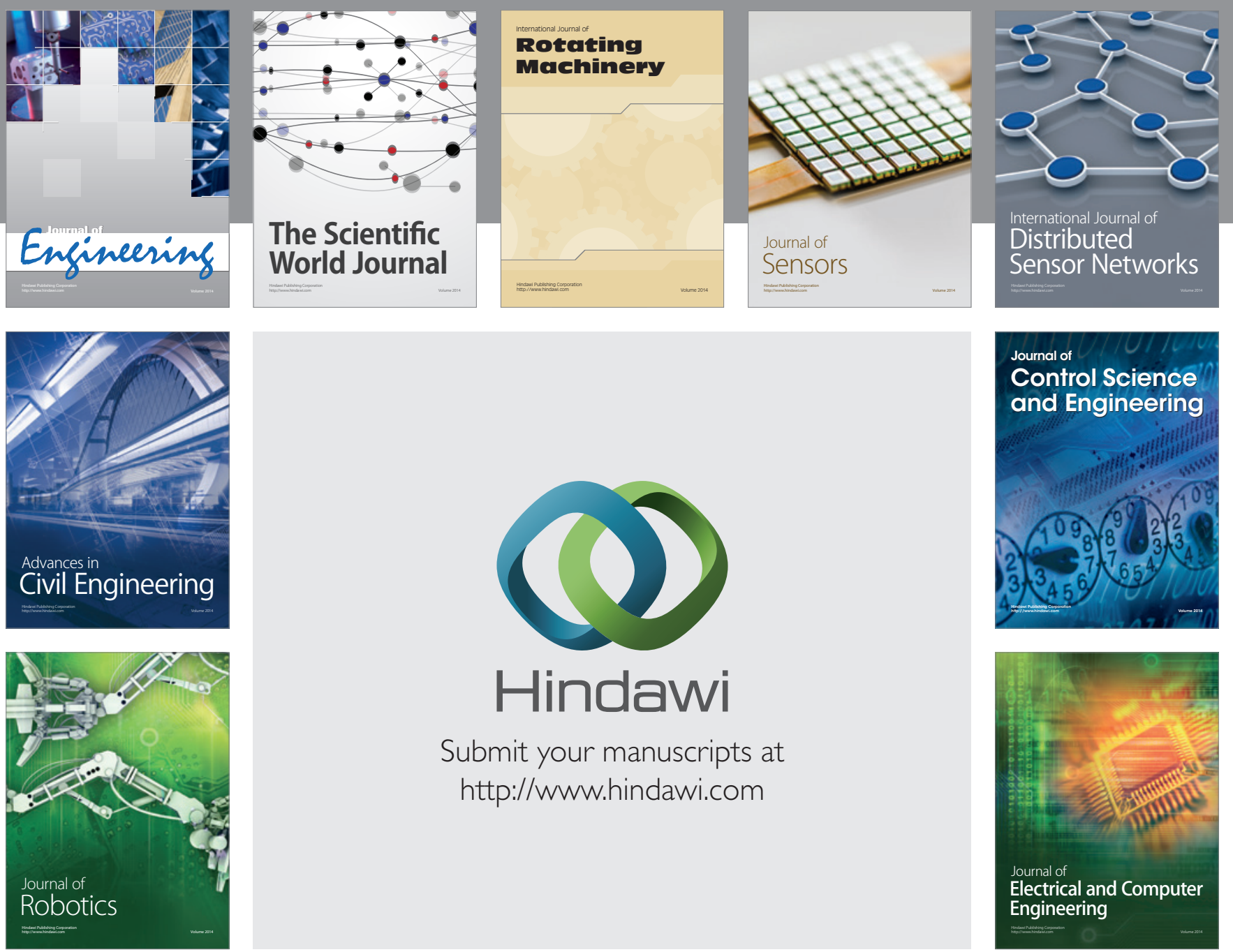

Submit your manuscripts at

http://www.hindawi.com
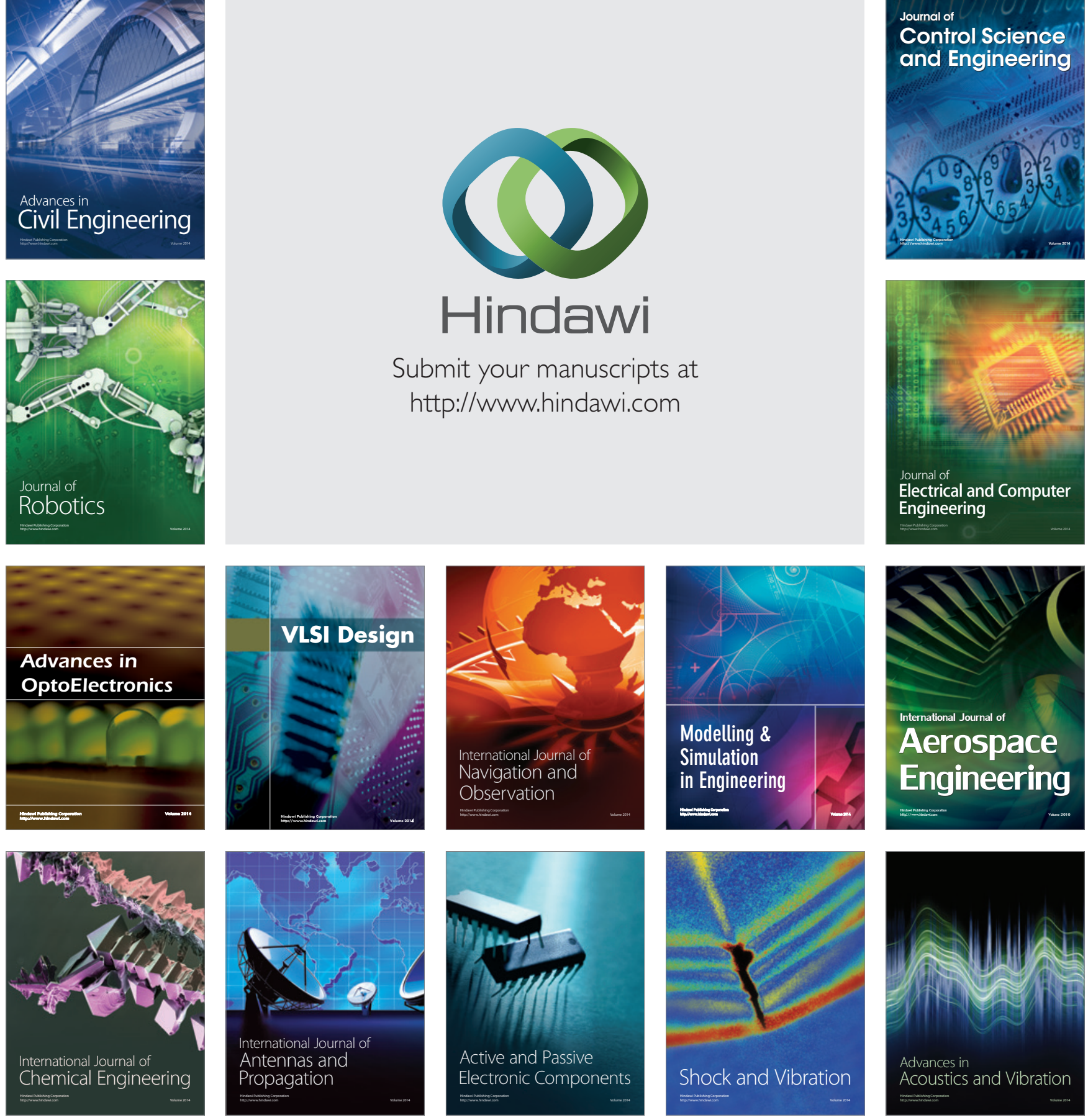